\title{
Long noncoding RNA TUG1 promotes renal cell carcinoma cell proliferation, migration and invasion by downregulating microRNA-196a
}

\author{
YANG YANG, DE-MING SUN, JUN-FENG YU, MAN ZHANG, \\ CHENG YI, RUI YANG, BAO-HUA DAN and AI-JUN LI
}

\begin{abstract}
Department of Urological Surgery, China Three Gorges University Affiliated Yichang City First People's Hospital, Yichang, Hubei 443000, P.R. China
\end{abstract}

Received January 10, 2017; Accepted December 14, 2017

DOI: $10.3892 / \mathrm{mmr} .2018 .9608$

\begin{abstract}
Long noncoding RNA taurine upregulated gene 1 (lncRNA TUG1) and microRNA-196a (miR-196a) have been reported to serve important roles in the development of renal cell carcinoma (RCC). However, their potential mechanisms have not been completely elucidated. The aim of the present study was to clarify the biological functions of lncRNA-TUG1 and miR-196a, in addition to investigating the interaction between lncRNA-TUG1 and microRNA-196a, providing a novel insight into RCC tumorigenesis. The present study comprised two parts. In the first part, IncRNA-TUG1 was confirmed as an oncogene, via reverse transcription-quantitative polymerase chain reaction (RT-qPCR) analysis, MTT assay, flow cytometry analysis, and migration and invasion assays. In the second part, the association between IncRNA-TUG1 and miR-196a, and the molecular mechanism, was illustrated via RT-qPCR analysis, MTT assay, dual luciferase reporter assay and western blotting. The results of the present study demonstrated that IncRNA-TUG1 was able to promote RCC cell proliferation, migration and invasion in vitro by suppressing miR-196a. Additionally, lncRNA-TUG1 achieved its biological functions by regulating the expression levels of RAC- $\alpha$ serine/threonine-protein kinase, mitogen-activated protein kinase and extracellular signal-regulated kinase via inhibition of miR-196a. In conclusion, the present findings proposed a novel potential therapeutic target, the IncRNA-TUG1-miR-196a axis, which may be applicable to the treatment of RCC.
\end{abstract}

Correspondence to: Dr Ai-Jun Li, Department of Urological Surgery, China Three Gorges University Affiliated Yichang City First People's Hospital, 2 Jiefang Road, Yichang, Hubei 443000, P.R. China E-mail: liaijun198080@163.com

Abbreviations: lncRNA, long noncoding RNA; TUG1, taurine upregulated gene 1; miR-196a, microRNA-196a; RCC, renal cell carcinoma

Key words: lncRNA TUG1, miR-196a, RCC

\section{Introduction}

Renal cell carcinoma ( $\mathrm{RCC}$ ) is the most common lethal urological carcinoma in adults, with an increasing global incidence rate (1). At present, surgery, chemotherapy and radiotherapy are the most frequently used therapies for $\operatorname{RCC}(2,3)$. Increasing studies have focused on minimizing damage while achieving total destruction of the tumor, to achieve decreased recurrence and a better prognosis for patients with RCC (4). Therefore, the present study was performed to examine novel therapeutic targets for RCC.

Long noncoding RNA (lncRNA) is a class of noncoding transcribed RNA molecules with $>200$ bases. Emerging evidence has demonstrated that lncRNAs appear to be associated with a number of malignancies, and act as tumor oncogenes or suppressors, which regulate gene expression through regulation of transcription, post-transcription, genomic imprinting and chromatin modification (5-7). MicroRNAs (miRs) are a class of small noncoding RNAs of 17-22 nucleotides in length (8). Previous studies have demonstrated that miRs regulate various cellular processes, and certain miRs are aberrantly expressed in cancer cells, acting as oncogenes or tumor suppressors by targeting mRNAs, and function as an RNA-induced silencing complex (9-12). Therefore, lncRNAs and miRs are potential diagnostic and prognostic biomarkers of cancer in clinical practice.

lncRNA taurine upregulated gene 1 (TUG1), an lncRNA located at chromosome $22 \mathrm{q} 12$, was first identified as a part of photoreceptors and during retinal development in mouse retinal cells (13). In further studies, TUG1 was considered to be involved in regulating carcinogenesis in a number of malignant tumors, and TUG1 has been reported to be a predictive biomarker of RCC, while the specific biological function and mechanism of lncRNA-TUG1 in RCC remains unclear (14-16). It has additionally been reported that miR-196a may be aberrantly expressed in numerous types of cancer, including RCC; certain studies reported that miR-196a expression is downregulated in RCC cells, although its function and mechanism remains to be elucidated (17-20).

In the present study, the expression level of IncRNA-TUG1 and microRNA-196a was detected in RCC cells, and 
the biological activity, and functional mechanisms, of lncRNA-TUG1 and microRNA-196a in regulating RCC cell proliferation, migration and invasion were investigated in vitro.

\section{Materials and methods}

Cell culture. Human renal cell carcinoma (RCC) cell lines 786-O, ACHN, A704, A498 and normal kidney cell line HK-2 were purchased from the Type Culture Collection of the Chinese Academy of Medical Sciences (Shanghai, China). All the cell lines were cultured in Dulbecco's modified Eagle's medium (DMEM) with 10\% fetal bovine serum (FBS), $1 \% 100 \mu \mathrm{l} / \mathrm{ml}$ penicillin-streptomycin and $1 \% 100 \mathrm{U} / \mathrm{ml}$ glutamine (all Kaiji, Nanjing, China) and grown in a humidified incubator containing $5 \% \mathrm{CO}_{2}$ at $37^{\circ} \mathrm{C}$.

$R N A$ extraction and reverse transcription-quantitative polymerase chain reaction $(R T-q P C R)$ analyses. Total RNA from cultured cells was extracted using TRIzol reagent (Invitrogen; Thermo Fisher Scientific, Inc., Waltham, MA, USA), according to the manufacturer's protocol, and corresponding cDNA was transcribed from total RNA using primers and a Revert Aid First Strand cDNA Synthesis kit (Thermo Fisher Scientific, Inc.), according to the manufacturer's instructions. qPCR analysis was performed to detect relevant gene expression using a SYBR-Green PCR Master Mix kit (Takara Biotechnology Co., Ltd., Dalian, China) and the ABI 7500 Real-Time PCR System (Applied Biosystems; Thermo Fisher Scientific, Inc.), according to the manufacturer's protocol. GAPDH was designated as the internal control. The primer sequences for GAPDH were forward, 5'-GTGTCTGAGCGA TGTGGCT-3' and reverse, 5'-GGATTTGGTCGTATTGGG C-3'; the primer sequences for forward, IncRNA-TUG1 were 5'-TAGCAGTTCCCCAATCCTTG-3' and reverse, 5'-CAC AAATTCCCATCATTCCC-3'; and the primer sequences for miR-196a were forward, 5'-TGTCTCACTGCACCTTCA-3' and reverse, 5'-GACTTCCCAACGCCTCCT-3'. The PCR cycling conditions were as follows: $95^{\circ} \mathrm{C}$ for $5 \mathrm{~min}$, followed by 45 cycles of $95^{\circ} \mathrm{C}$ for $10 \mathrm{sec}$ and $60^{\circ} \mathrm{C}$ for $30 \mathrm{sec}$. The gene expression was normalized to GAPDH. All results were repeated three times and expressed as the mean \pm standard deviation of three independent experiments. Results were analyzed using the $2^{-\Delta \Delta \mathrm{Cq}}$ method (21).

Lentiviral vector construction. Packaging vectors carrying green fluorescent protein (GFP), lncRNA-TUG1, and short hairpin (sh)RNA-TUG1 were constructed. Then the packaging vectors (pCMV-VSVG, pMDLg/pRRE and pRSV-REV) and the lentiviral vectors (all Shanghai GenePharma, Co., Ltd., Shanghai, China) were co-transfected into HK-2 cells $\left(1 \times 10^{5}\right.$ cells/well) using Lipofectamine ${ }^{\circledR} 2000$ (Invitrogen; Thermo Fisher Scientific, Inc.), according to the manufacturer's protocol. Following $8 \mathrm{~h}$ transfection, the medium was replaced with fresh DMEM containing 10\% FBS and the cells were cultured for a further $48 \mathrm{~h}$. Subsequently, the cell culture supernatant containing vectors was collected, centrifuged at $4,000 \mathrm{x} \mathrm{g}$ for $10 \mathrm{~min}$ at room temperature and filtered through a $0.45-\mu \mathrm{m}$ filter. The lentiviral vectors, which are referred to as lenti-GFP, lenti-lncRNA-TUG1 or lenti-shTUG1, were stored at $-80^{\circ} \mathrm{C}$ until further use.
Transfection. RCC $786-\mathrm{O}$ cells $\left(1 \times 10^{5}\right.$ cells/well $)$ were transfected with $20 \mathrm{nM}$ lenti-GFP, lenti-lncRNA-TUG1 or lenti-shTUG1 in $8 \mu \mathrm{g} / \mathrm{ml}$ polybrene (Sigma-Aldrich; Merck KGaA, Darmstadt, Germany). Subsequently, G418 (Life Technologies; Thermo Fisher Scientific, Inc.; $0.8 \mathrm{mg} / \mathrm{ml}$ ) was applied to screen and establish the stable expression of transfected genes. RCC 786-O cells $\left(2 \times 10^{5}\right.$ cells/well) were transfected with $10 \mathrm{nM}$ negative control mimics (5'-UUC UCCGAACGUGUCACGUTT-3'), miR-196a mimic (5'-UAG GUAGUUUCAUGUUGUUGGG-3'), or miR-inhibitor mimics (5'-TACGGAGGTGGAGTGGGTGTGG-3'; Shanghai GenePharma Co.,Ltd.) using Lipofectamine ${ }^{\circledR} 2000$ (Invitrogen; Thermo Fisher Scientific, Inc.) for $72 \mathrm{~h}$.

MTT assay. MTT assays were used to detect the proliferative ability of RCC 786-O cells with or without transfection. A total of $2 \times 10^{3}$ cells were seeded in a 96-well plate and incubated with $0.5 \mathrm{mg} / \mathrm{ml}$ MTT solution (Sigma-Aldrich; Merck KGaA) for $4 \mathrm{~h}$ at $37^{\circ} \mathrm{C}$. Subsequently, the original culture medium was discarded and $100 \mu$ ldimethyl sulfoxide solution (Sigma-Aldrich; Merck $\mathrm{KGaA}$ ) was added to each well. The absorbance was detected at a wavelength of $490 \mathrm{~nm}$ using a Tecan plate reader (Bio-Rad Laboratories, Inc., Hercules, CA, USA).

Flow cytometry analysis. Apoptotic rates of RCC 786-O cells with or without transfection of lenti-GFP, lenti-lncRNA-TUG1 or lenti-shTUG1 were analyzed using an Annexin V-fluorescein isothiocyanate (FITC)/propidium iodide (PI) apoptosis detection kit (BioVision, Inc., Milpitas, CA, USA), as per the manufacturer's protocol. Briefly, $5 \times 10^{5}$ cells were harvested and washed with PBS twice, prior to being resuspended in $500 \mu \mathrm{l}$ binding buffer and incubated with $5 \mu \mathrm{l}$ Annexin V-FITC, and $5 \mu \mathrm{l}$ PI, for $10 \mathrm{~min}$ at room temperature in the dark. The apoptotic rates of 786-O cells were examined using a flow cytometer (BD Biosciences, Franklin Lakes, NJ, USA) and BD CellQuest ${ }^{\mathrm{TM}}$ Acquisition software version 2.9 (BD Biosciences). Cells were distinguished as viable, early apoptotic, apoptotic or dead.

Migration and invasion assays. Transwell assays were performed to determine the migratory and invasive ability of RCC 786-O cells transfected with lenti-lncRNA-TUG1 or lenti-shTUG1. To assess cell migration, $1 \times 10^{5} 786-\mathrm{O}$ cells in serum-free RPMI 1640 medium (Kaiji) were seeded onto Transwell inserts (pore size, $8 \mu \mathrm{m}$; Costar; Corning Incorporated, Corning, NY, USA). Subsequently, $600 \mu 1$ RPMI medium supplemented with 10\% FBS was added to the lower chambers. Cells were incubated for $24 \mathrm{~h}$ and non-migrated cells on the upper surface were removed. The inserts were washed with PBS, fixed with $4 \%$ methanol for $20 \mathrm{~min}$ at $37^{\circ} \mathrm{C}$ and stained with $0.1 \%$ crystal violet for $5 \mathrm{~min}$ at room temperature. The number of migratory cells was counted under a light microscope (magnification, x200; Nikon Corporation, Tokyo, Japan). To assess invasion, the same protocol for cell migration was used; however, the Transwell insert membranes were pre-coated with a layer of 200-300 $\mu \mathrm{g} / \mathrm{ml}$ Matrigel (BD Biosciences) for $6 \mathrm{~h}$ at $37^{\circ} \mathrm{C}$.

Western blot analysis. Western blot analysis was used to assess the expression levels of relevant proteins. RCC 786-O cells were 


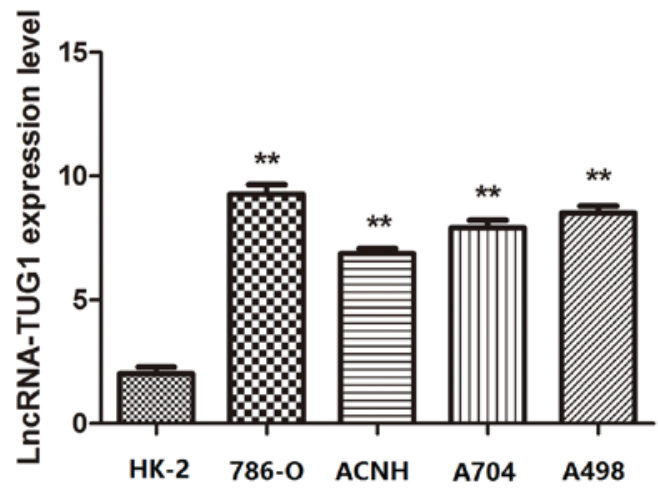

Figure 1. Expression level of lncRNA-TUG1 increases in human RCC cell lines. The expression level of lncRNA-TUG1 in the normal kidney cell line HK-2 and RCC cell lines 786-O, ACHN, A704 and A498 was detected by reverse transcription-quantitative polymerase chain reaction and quantified using the comparative $2^{-\Delta \Delta C q}$ method. ${ }^{* *} \mathrm{P}<0.01$ vs. HK-2. IncRNA long noncoding RNA; TUG1, taurine upregulated gene 1; RCC, renal cell carcinoma.

harvested in radioimmunoprecipitation assay lysis buffer $(50 \mathrm{mM}$ Tris/HCl, pH 7.4; 150 mM NaCl; 1\% NP-40; and 0.1\% SDS) containing a protease inhibitor cocktail (Sigma-Aldrich; Merck $\mathrm{KGaA}$ ). Supernatants were collected by centrifugation at $6,000 \mathrm{x} \mathrm{g}$ for $5 \mathrm{~min}$ at $4^{\circ} \mathrm{C}$ and the protein concentration was detected using a Bio-Rad Protein Assay kit (cat. no. 5000002; Bio-Rad Laboratories, Inc.). A total of $30 \mu \mathrm{g}$ protein was separated on a $10 \%$ SDS-PAGE gel and transferred to polyvinylidene difluoride membranes (EMD Millipore, Billerica, MA, USA). After blocking in Tris-buffered saline containing 5\% nonfat milk for $2 \mathrm{~h}$ at room temperature, membranes were incubated with primary antibodies at $4^{\circ} \mathrm{C}$ overnight, including anti-phosphorylated (p) RAC- $\alpha$ serine/threonine-protein kinase (1:1,000; cat. no. sc-16646; Santa Cruz Biotechnology, Inc., Dallas, TX, USA), anti-AKT (1:1,000; cat. no. ab38449; Abcam, Cambridge, UK), anti-p-extracellular signal-regulated kinase (ERK) antibody (1:1,000; cat. no. sc-7383, Santa Cruz Biotechnology, Inc.), anti-ERK antibody (1:1,000; cat. no. ab32537), anti-mitogen-activated protein kinase 8 (JNK1) antibody (1:1,000; cat. no. ab199380), anti-mitogen-activated protein kinase 9 (JNK2) antibody (1:1,000; cat. no. ab178953) and anti-GAPDH antibody (1:4,000; cat. no. ab9485; all Abcam). Membranes were subsequently washed three times in PBS containing $0.1 \%$ (v/v) Tween-20 (Invitrogen; Thermo Fisher Scientific, Inc.) and incubated with goat anti-rabbit (1:5,000; cat. no. ab7090) or anti (1:5,000; cat. no. ab8226; both Abcam) horseradish peroxidase-conjugated IgG secondary antibodies for $1 \mathrm{~h}$ at room temperature. The enhanced chemiluminescence (ECL) substrate kit and the enhanced chemiluminescence detection system (Amersham; GE Healthcare, Chicago, IL, USA) were used to develop and detect protein bands. BandScan software version 5.0 (Glyko, Inc., Novato, CA, USA) was used for densitometric analysis following western blotting.

Dual luciferase reporter assay. Wild type TUG1 gene with miR-196a binding sites or mutant TUG1 gene with target site deletions were constructed, amplified and cloned into the pRL-TK plasmid (Promega Corporation, Madison, WI, USA) vector. A total of $2 \times 10^{4} 786-\mathrm{O}$ cells/well were seeded into 96-well plates. Cells were co-transfected with the constructed plasmids using Lipofectamine ${ }^{\circledR} 2000$, and Renilla luciferase was used as an internal control. The cells were harvested $48 \mathrm{~h}$ following transfection and the luciferase activities were detected by a Dual-Luciferase Reporter Assay kit (Promega Corporation), as per instructions provided by the manufacturer.

Statistical analysis. Statistical analyses were performed using GraphPad Prism version 6.0 (GraphPad Software, Inc., La Jolla, CA, USA). Statistical significance was tested by Student's t test or one-way analysis of variance followed by the Dunnett's post hoc test. All results are expressed as the mean \pm standard deviation. $\mathrm{P}<0.05$ was considered to indicate a statistically significant difference. All experiments were repeated at least three times.

\section{Results}

lncRNA-TUG1 expression is increased in human RCC cell lines. The present study examined the mRNA expression level of lncRNA-TUG1 in five cell lines, including the normal kidney cell line HK-2, and RCC cell lines 786-O, ACHN, A704 and A498, using RT-qPCR. The results demonstrated that the expression level of lncRNA-TUG1 was significantly increased in RCC cells compared with HK-2 cells (Fig. 1). Subsequently, the RCC cell lines 786-O was selected for further experiments, as it expressed the highest TUG1 mRNA level of the four RCC cell lines.

IncRNA-TUG1 promotes RCC cell proliferation, migration and invasion in vitro. To investigate the biological role of lncRNA-TUG1 in RCC, the present study analyzed the proliferative, migratory and invasive ability of RCC 786-O cells with or without transfection of lenti-GFP, lenti-lncRNA-TUG1 or lenti-shTUG1. RT-qPCR was used to determine the expression level of IncRNA-TUG1, from which it was demonstrated that lncRNA-TUG1 expression was significantly elevated in the lenti-lncRNA-TUG1 group, while significantly decreased in the lenti-shTUG1 group, compared with the control groups (Fig. 2A). An MTT assay and flow cytometry analysis were used to measure the effect of 1ncRNA-TUG1 on the cell viability and apoptosis rate of RCC cells. The results demonstrated that IncRNA-TUG1-overexpressing cells exhibited improved cell viability and a lower apoptosis rate, while lncRNA-TUG1-downregulated cells exhibited a poorer proliferative ability and a higher apoptosis rate, compared with the two control groups (Fig. 2B-D). Migration and invasion assays were performed to evaluate the impact of IncRNA-TUG1 on RCC migration and invasion. From these assays it was revealed that the migratory and invasive cell numbers were significantly increased in the lenti-lncRNA-TUG1 group, while significantly decreased in the lenti-shTUG1 group compared with the control (Fig. 2E-H). These results suggested that lncRNA-TUG1 may serve a tumor-promoting role in the development of RCC.

IncRNA-TUG1 downregulates miR-196a in human RCC cells. To elucidate the functional mechanisms of lncRNA-TUG1 as an oncogene, the present study detected the expression level of miR-196a by RT-qPCR in five cell lines, including the normal kidney cell line HK-2, and the RCC cell lines 786-O, ACHN, A704 and A498. The results demonstrated that miR-196a expression was significantly decreased in RCC cells compared 
A

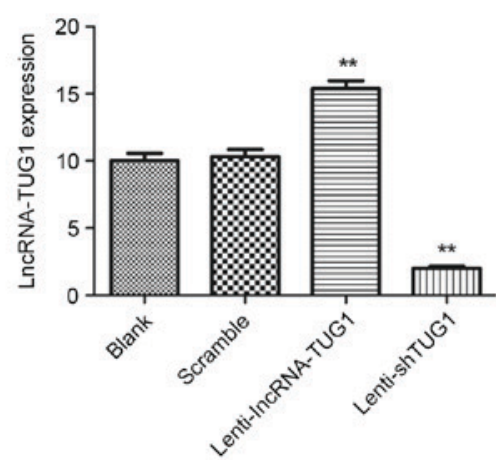

C
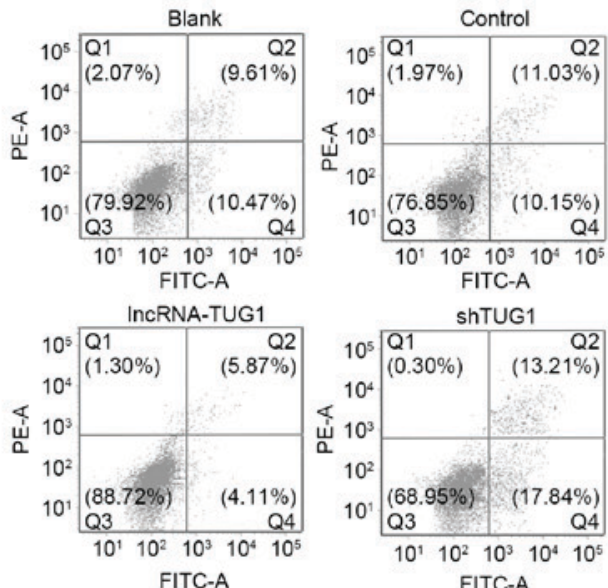

Blank

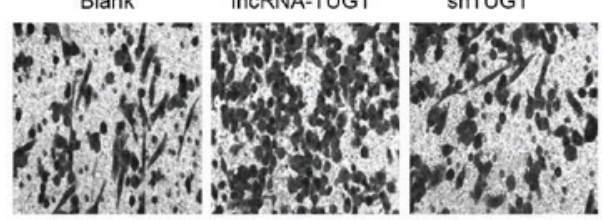

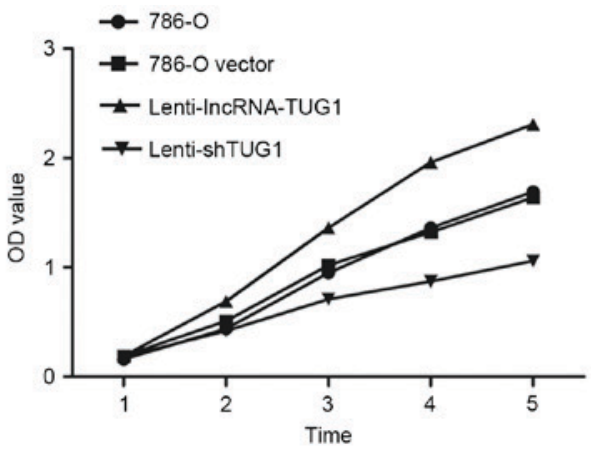

D
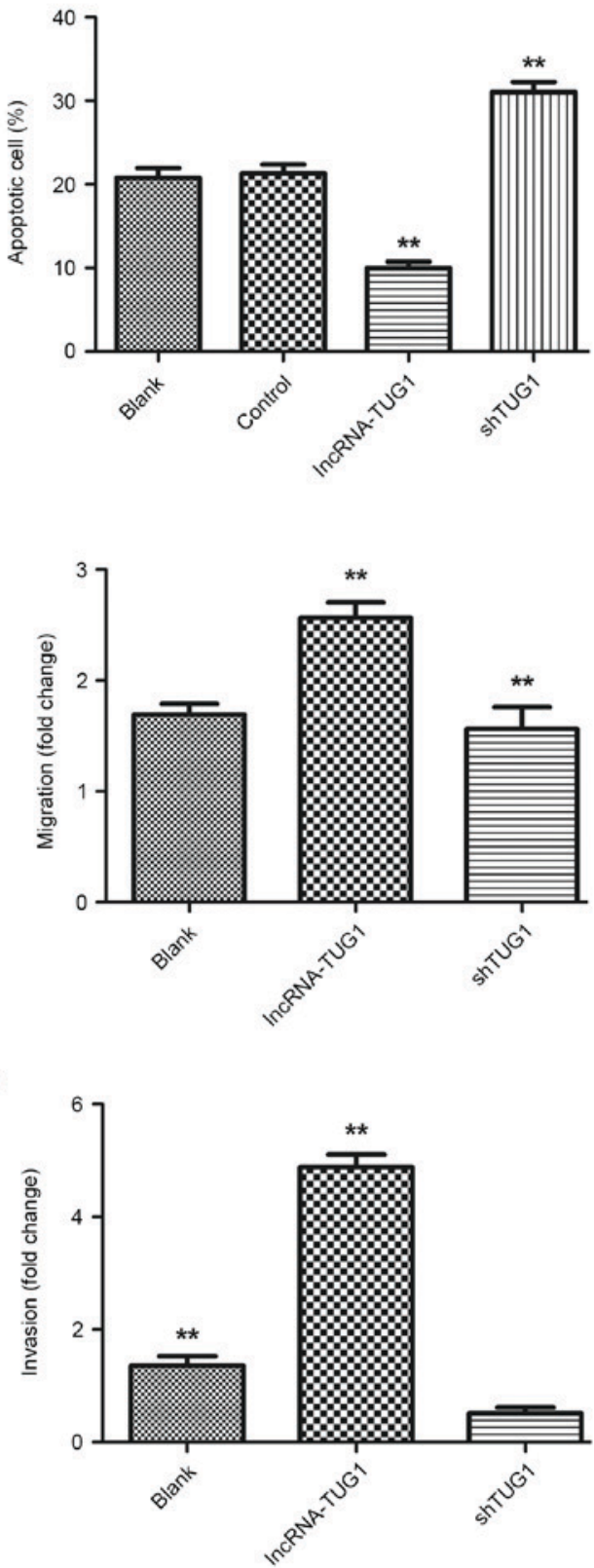

Figure 2. IncRNA-TUG1 promotes RCC cell proliferation, migration and invasion. (A) The expression level of lncRNA-TUG1 was detected by reverse transcription-quantitative polymerase chain reaction in four groups, including a blank control group, negative control group, lenti-lncRNA-TUG1 group and lenti-shTUG1 group (value of $2^{-\Delta \triangle C q}$ ). (B) The cell viability of RCC 786-O cells per day was measured by MTT assays with or without transfection of control vectors, lenti-IncRNA-TUG1 or lenti-shTUG1. The apoptosis rate of RCC 786-O cells was determined by (C) flow cytometry analysis with or without transfection of control vectors, lenti-lncRNA-TUG1 or lenti-shTUG1, and (D) the results were quantified. The migratory ability of RCC 786-O cells was measured by (E) migration assay, prior to and following transfection with lenti-lncRNA-TUG1 or lenti-shTUG1. Migratory cells were stained with crystal violet solution (magnification, x200), and (F) the quantification of the relative migrated cell number is presented in the graph. The invasive ability of RCC 786-O cells was detected by (G) Matrigel invasion assay prior to and following transfection with lenti-lncRNA-TUG1 or lenti-shTUG1. Invasive cells were stained with crystal violet solution (magnification, $\mathrm{x} 200)$, and $(\mathrm{H})$ the quantification of the relative invaded cell number is presented in the graph. ${ }^{* *} \mathrm{P}<0.01 \mathrm{vs.} \mathrm{Blank}$. IncRNA, long noncoding RNA; TUG1, taurine upregulated gene 1; sh, short hairpin; RCC, renal cell carcinoma; PE, phycoerythrin; FITC, fluorescein isothiocyanate; OD, optical density. 
A

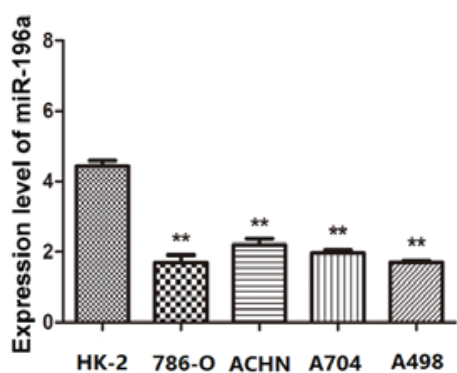

B

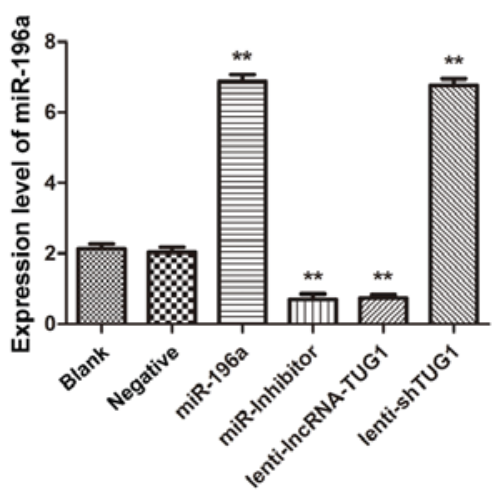

C

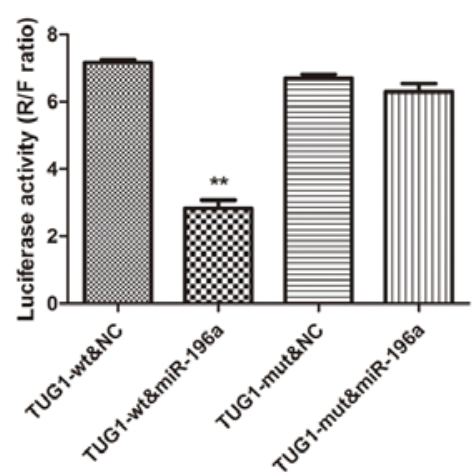

Figure 3. miR-196a is downregulated in RCC cells by lncRNA-TUG1. (A) The RT-qPCR was used to detect the mRNA expression level of miR-196a in RCC cell lines and the normal kidney cell line HK-2, and the results were analyzed using the comparative $2^{-\Delta \Delta C q}$ method. ${ }^{* *} \mathrm{P}<0.01$ vs. $\mathrm{HK}-2$. (B) The mRNA expression level of miR-196a was measured by RT-qPCR in RCC 786-O cells transfected with lncRNA-TUG1, shTHG1, miR196a or miR-inhibitor. ${ }^{* *}$ P<0.01 vs. Blank. (C) The luciferase reporter assay was used to detect the luciferase activity of lncRNA-TUG1 in HK-2 cells co-transfected with lncRNA-TUG1 (wt or mut) and miR-196a mimics or control mimics. ${ }^{* *} \mathrm{P}<0.01$ vs. TUG1-wt\&NC. RT-qPCR, reverse transcription-quantitative polymerase chain reaction; RCC, renal cell carcinoma; miR, microRNA; lncRNA, long noncoding RNA; TUG1, taurine upregulated gene 1; wt, wild type; mut, mutant; NC, negative control.

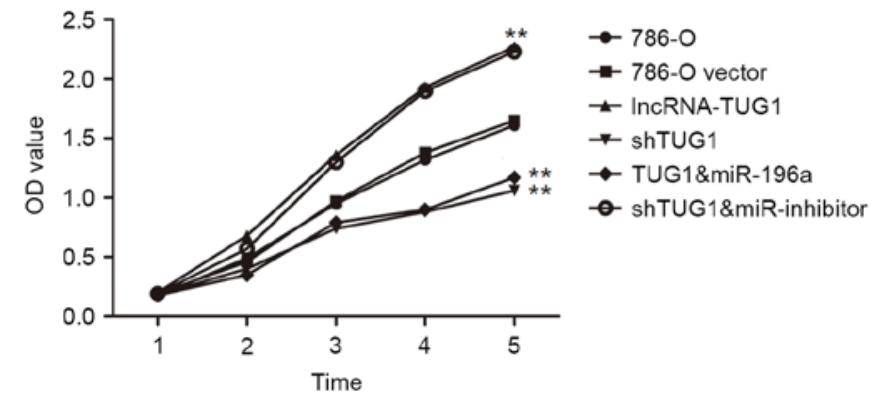

Figure 4.IncRNA-TUG1 promotes the proliferation of RCC cells by suppressing miR-196a. The cell viability of RCC 786-O cells was determined without transfection or following transfection with scramble vectors, lncRNA-TUG1, shTUG1, IncRNA-TUG1 and miR-196a, or shTUG1 and miR-inhibitor using an MTT assay. miR, microRNA; lncRNA, long noncoding RNA; TUG1, taurine upregulated gene 1; RCC, renal cell carcinoma; OD, optical density. ${ }^{* *} \mathrm{P}<0.01$ vs. $786-\mathrm{O}$.

with HK-2 cells (Fig. 3A). To determine whether the low expression level of miR-196a was relevant to the overexpressed lncRNA-TUG1 in RCC cell lines, the present study determined the miR-196a expression level following transfection. The results demonstrated that the expression level of miR-196a significantly increased following transfection with miR-196a or lenti-shTUG1 compared with the controls, while it significantly decreased following transfection with miR-inhibitor or lenti-lncRNA-TUG1 (Fig. 3B). From these results, it was further hypothesized that miR-196a may be involved in the lncRNA-TUG1 tumor-promoting function in RCC via a binding interaction. Therefore, a luciferase reporter assay was performed to verify this hypothesis, which demonstrated that luciferase activity was significantly decreased following co-transfection of miR-196a mimics and wild-type lncRNA-TUG1 (Fig. 3C). The results of the present study indicated that there may be a binding site between IncRNA-TUG1 and miR-196a, through which miR-196a may serve an important role in the biological function of lncRNA-TUG1 in RCC.

IncRNA-TUG1 promotes RCC cell proliferation via suppression of $m i R-196 a$. Cell viability was determined using an
MTT assay following transfection of RCC 786-O cells with lncRNA-TUG1 or shTUG1, or co-transfection with lncRNA-TUG1 and miR-196a, shTUG1 and miR-inhibitor. Consistent with the above results, it was observed that cells transfected with lncRNA-TUG1 or shTUG1 had markedly better or poorer cell viability, respectively, compared with the controls. Furthermore, this decrease or increase in cell viability was able to be reversed by co-transfection with miR-inhibitor or miR-196a (Fig. 4). In general, these results confirmed the above findings, which revealed that IncRNA-TUG1 promoted RCC cell proliferation by suppressing miR-196a.

IncRNA-TUG1 regulates $J N K, p-E R K$ and $p$-AKT expression in human RCC cells. In order to elucidate the molecular mechanisms underlying the tumor-promoting function of lncRNA-TUG1 in RCC, western blotting was used to detect relevant protein expression levels, including JNK, p-ERK and p-AKT, in RCC 786-O cells. It was observed that the expression of $\mathrm{p}-\mathrm{AKT}$ was upregulated when cells were transfected with lenti-lncRNA-TUG1, which was reversed following co-transfection with miR-196. By contrast, the expression of p-JNK and p-ERK exhibited a corresponding upregulation when RCC 786-O cells were transfected with lenti-shTUG1, which was reversed by co-transfection with miR-inhibitor (Fig. 5). These data indicated that IncRNA-TUG1 may regulate proliferation-associated proteins by suppressing miR-196a, thereby promoting tumorigenesis in RCC.

\section{Discussion}

With the number of patients with RCC increasing every year worldwide, there is an urgent requirement for more efficient and precise therapies (2,3). At present, a number of studies have reported that the abnormal expression of proteins is involved in regulating various RCC cell pathological processes, including proliferation, migration and invasion $(22,23)$. Simultaneously, studies have detected noncoding RNAs in RCC cell genomes, which are considered to be important elements in modifying protein 

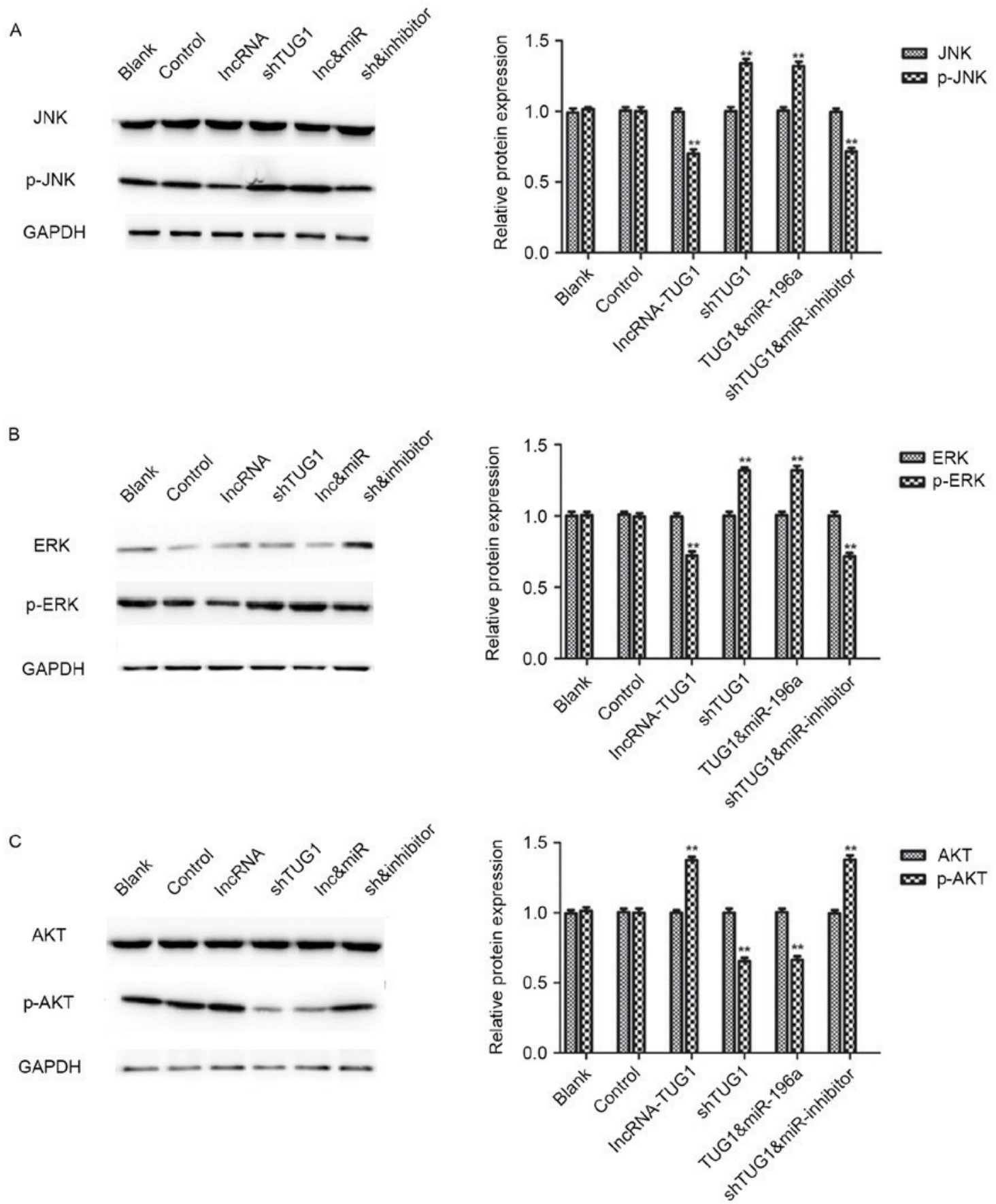

Figure 5. 1ncRNA-TUG1 regulates protein expression in RCC 786-O cells by suppressing miR-196a. (A) Western blotting was used to detect the expression level of JNK in RCC 786-O cells. The results demonstrated that lncRNA-TUG1 downregulated p-JNK expression by suppressing miR-196a. (B) Western blotting was used to detect the expression level of ERK in RCC 786-O cells. The results demonstrated that lncRNA-TUG1 downregulated p-ERK expression by suppressing miR-196a. (C) Western blotting was used to detect the expression level of AKT in RCC 786-O cells. The results demonstrated that lncRNA-TUG1 upregulated p-AKT expression by suppressing miR-196a. ${ }^{* *} \mathrm{P}<0.01$ vs. Control. IncRNA, long noncoding RNA; TUG1, taurine upregulated gene 1; p, phosphorylated; RCC, renal cell carcinoma; JNK, mitogen-activated protein kinase; ERK, extracellular signal-regulated kinase; AKT, RAC- $\alpha$ serine/threonine-protein kinase; miR, microRNA.

expression $(5,22,23)$. Therefore, analyzing noncoding RNAs may be an effective way to examine novel potential biomarkers and their molecular mechanisms in the development of RCC.

Noncoding RNAs are classified into two classes: Small noncoding RNAs (miRs) and lncRNAs. miRs target at mRNAs at the post-transcriptional stage, resulting in mRNA degradation $(24,25)$. IncRNAs have been reported to serve an important role in gene regulation in multiple ways, including epigenetic, transcriptional, posttranscriptional and translational functions $(26,27)$. miRs and lncRNAs are considered to be associated with the occurrence of numerous malignant diseases (28).

In present study, it was observed that the expression level of lncRNA-TUG1 was increased in human RCC cell lines. Therefore, lentiviral vectors were constructed that carried IncRNA-TUG1, shTUG1 or scramble genes, to artificially regulate lncRNA-TUG1 expression levels. By introducing 
lentiviral vectors to RCC 786-O cells, the present study determined that IncRNA-TUG1 was able to promote RCC cell proliferation, migration and invasion in vitro. It was demonstrated that lncRNA-TUG1 acts as an oncogene. A similar finding was reported in previous studies. Han et al (29) reported that IncRNA-TUG1 was overexpressed in urothelial carcinomas, and that the high expression level was predictive for high grade and stage; additionally, downregulated lncRNA-TUG1 led to proliferation inhibition and a decrease in apoptosis. In another study, Zhang et al (16) reported that lncRNA-TUG1 was overexpressed in RCC, and silencing of it led to inhibition of proliferation, migration and invasion, in addition to the promotion of apoptosis.

In further experiments, the present study demonstrated that miR-196a expression was significantly decreased in RCC cell lines. Therefore, miR-196a, miR-inhibitor or scramble mimics were transfected into RCC 786-O cells. Via co-transfection of lentiviral vectors and microRNA mimics, an association between the expression levels of lncRNA-TUG1 and miR-196a was demonstrated. The dual luciferase reporter assay further confirmed that lncRNA-TUG1 and miR-196a had a binding site. Re-examination of cell viability revealed that IncRNA-TUG1 achieved its functions in RCC cells by suppressing miR-196a. The present study was the first, to the best of our knowledge, to discuss the association between lncRNA-TUG1 and miR-196a, and their functions in RCC. JNK and p-ERK are widely accepted as proteins that promote cell death. p-AKT is regarded as a regulator of cell survival (30,31). All of these proteins have been reported to serve important roles in cancer cell proliferation, growth and survival $(32,33)$. In the present study, the expression of the aforementioned proteins was determined post-transfection, and it was observed that the lncRNA-TUG1/miR-196a axis may regulate these proteins to interfere with RCC cell proliferation.

In conclusion, although there remain further details to be elucidated with respect to the lncRNA-TUG1/miRNA-196a axis, the present study clarified the expression levels of IncRNA-TUG1 and miR-196a in RCC cells. In addition, the present study confirmed the function of lncRNA-TUG1 as an oncogene, which achieves its biological functions in RCC via regulation of protein expression by suppressing miR-196a. The results of the present study revealed a novel oncogenic pathway in the development of RCC, which may be a promising therapeutic target in the future.

\section{Acknowledgements}

The authors of the present study would like to thank the Basic Medical Research Center of Three Gorges University (Yichang, China) for technical assistance.

\section{Funding}

Not applicable.

\section{Availability of data and materials}

The datasets used and/or analyzed during the current study are available from the corresponding author on reasonable request.

\section{Authors' contributions}

YY, A-JL and D-MS designed the study. J-FY and MZ analyzed the data. CY, B-HD and RY performed the experiments. A-JL and YY wrote the manuscript. All authors read and approved the final manuscript.

\section{Ethics approval and consent to participate}

Not applicable.

\section{Patient consent for publication}

Not applicable.

\section{Competing interests}

The authors declare that they have no competing interests.

\section{References}

1. Ljungberg B, Campbell SC, Choi HY, Jacqmin D, Lee JE, Weikert S and Kiemeney LA: The epidemiology of renal cell carcinoma. Eur Urol 60: 615-621, 2011.

2. Castro A Jr, Jenkins LC, Salas N, Lorber G and Leveillee RJ: Ablative therapies for small renal tumours. Nat Rev Urol 10: 284-291, 2013.

3. Jonasch E, Gao J and Rathmell WK: Renal cell carcinoma. BMJ 349: g4797, 2014.

4. Lardas M, Stewart F, Scrimgeour D, Hofmann F, Marconi L, Dabestani S, Bex A, Volpe A, Canfield SE, Staehler M, et al: Systematic review of surgical management of nonmetastatic renal cell carcinoma with vena caval thrombus. Eur Urol 70: 265-280, 2016.

5. Sanpakit K, Triwatanawong $J$ and Sumboonnanonda A: Long-term outcome in pediatric renal tumor survivors: Experience of a single center. J Pediatr Hematol Oncol 35: 610-613, 2013.

6. Xu Y, Wang J, Qiu M, Xu L, Li M, Jiang F, Yin R and Xu L: Upregulation of the long noncoding RNA TUG1 promotes proliferation and migration of esophageal squamous cell carcinoma. Tumour Biol 36: 1643-1651, 2015.

7. Guttman M and Rinn JL: Modular regulatory principles of large non-coding RNAs. Nature 482: 339-346, 2012.

8. Ha M and Kim VN: Regulation of microRNA biogenesis. Nat Rev Mol Cell Biol 15: 509-524, 2014.

9. Zhou X, Wang X, Huang Z, Wang J, Zhu W, Shu Y and Liu P: Prognostic value of miR-21 in various cancers: An updating meta-analysis. PLoS One 9: e102413, 2014.

10. Croce CM: Causes and consequences of microRNA dysregulation in cancer. Nat Rev Genet 10: 704-714, 2009.

11. Esquela-Kerscher A and Slack FJ: Oncomirs-microRNAs with a role in cancer. Nat Rev Cancer 6: 259-269, 2006.

12. Lund E, Güttinger S, Calado A, Dahlberg JE and Kutay U: Nuclear export of microRNA precursors. Science 303: 95-98, 2004.

13. Young TL, Matsuda T and Cepko CL: The noncoding RNA taurine upregulated gene 1 is required for differentiation of the murine retina. Curr Biol 15: 501-512, 2005.

14. Zhang Q, Geng PL, Yin P, Wang XL, Jia JP and Yao J: Down-regulation of long non-coding RNA TUG1 inhibits osteosarcoma cell proliferation and promotes apoptosis. Asian Pac J Cancer Prev 14: 2311-2315, 2013.

15. Zhang EB, Yin DD, Sun M, Kong R, Liu XH, You LH, Han L, Xia R, Wang KM, Yang JS, et al: P53-regulated long non-coding RNA TUG1 affects cell proliferation in human non-small cell lung cancer, partly through epigenetically regulating HOXB7 expression. Cell Death Dis 5: e1243, 2014.

16. Zhang M, Lu W, Huang Y, Shi J, Wu X, Zhang X, Jiang R, Cai Z and Wu S: Downregulation of the long noncoding RNA TUG1 inhibits the proliferation, migration, invasion and promotes apoptosis of renal cell carcinoma. J Mol Histol 47: 421-428, 2016. 
17. Pan J, Li X, Wu W, Xue M, Hou H, Zhai W and Chen W: Long non-coding RNA UCA1 promotes cisplatin/gemcitabine resistance through CREB modulating miR-196a-5p in bladder cancer cells. Cancer Lett 382: 64-76, 2016.

18. Tsai MM, Wang CS, Tsai CY, Chen CY, Chi HC, Tseng YH, Chung PJ, Lin YH, Chung IH, Chen $\mathrm{CY}$ and Lin $\mathrm{KH}$ : MicroRNA-196a/-196b promote cell metastasis via negative regulation of radixin in human gastric cancer. Cancer Lett 351 222-231, 2014.

19. White NM, Khella HW, Grigull J, Adzovic S, Youssef YM, Honey RJ, Stewart R, Pace KT, Bjarnason GA, Jewett MA, et al: miRNA profiling in metastatic renal cell carcinoma reveals a tumour-suppressor effect for miR-215. Br J Cancer 105 1741-1749, 2011.

20. Li Y, Jin L, Chen D, Liu J, Su Z, Yang S, Gui Y, Mao X, Nie G and Lai Y: Tumor suppressive miR-196a is associated with cellular migration, proliferation and apoptosis in renal cell carcinoma. Mol Med Rep 14: 560-566, 2016.

21. Livak KJ and Schmittgen TD: Analysis of relative gene expression data using real-time quantitative PCR and the 2(-Delta Delta C(T)) method. Methods 25: 402-408, 2001.

22. Kurozumi A, Goto $\mathrm{Y}$, Okato A, Ichikawa $\mathrm{T}$ and Seki N: Aberrantly expressed microRNAs in bladder cancer and renal cell carcinoma. J Hum Genet 62: 49-56, 2017.

23. Shenouda SK and Alahari SK: MicroRNA function in cancer: Oncogene or a tumor suppressor? Cancer Metastasis Rev 28: 369-378, 2009.

24. Zamore PD and Haley B: Ribo-gnome: The big world of small RNAs. Science 309: 1519-1524, 2005.
25. Calin GA and Croce CM: MicroRNA signatures in human cancers. Nat Rev Cancer 6: 857-866, 2006.

26. Qiu MT, Hu JW, Yin R and Xu L: Long noncoding RNA: An emerging paradigm of cancer research. Tumour Biol 34: 613-620, 2013.

27. Eades G, Zhang YS, Li QL, Xia JX, Yao Y and Zhou Q: Long non-coding RNAs in stem cells and cancer. World J Clin Oncol 5: 134-141, 2014.

28. Liz J and Esteller M: LncRNAs and microRNAs with a role in cancer development. Biochim Biophys Acta 1859: 169-176, 2016.

29. Han Y, Liu Y, Gui Y and Cai Z: Long intergenic non-coding RNA TUG1 is overexpressed in urothelial carcinoma of the bladder. J Surg Oncol 107: 555-559, 2013.

30. Vacotto M, Coso O and Fiszer de Plazas S: Programmed cell death and differential JNK, p38 and ERK response in a prenatal acute hypoxic hypoxia model. Neurochem Int 52: 857-863, 2008.

31. Chen CY, Weng YH, Chien KY, Lin KJ, Yeh TH, Cheng YP, Lu CS and Wang HL: (G2019S) LRRK2 activates MKK4-JNK pathway and causes degeneration of SN dopaminergic neurons in a transgenic mouse model of PD. Cell Death Differ 19: 1623-1633, 2012.

32. Wong CH, Iskandar KB, Yadav SK, Hirpara JL, Loh T and Pervaiz S: Correction: Simultaneous induction of non-canonical autophagy and apoptosis in cancer cells by ROS-dependent ERK and JNK activation. PLoS One 11: e0159352, 2016.

33. Osaki M, Oshimura M and Ito H: PI3K-Akt pathway: Its functions and alterations in human cancer. Apoptosis 9: 667-676, 2004. 